\title{
O uso de Lopinavir e Ritonavir como alternativa terapêutica para a COVID-19
}

\author{
The use of Lopinavir and Ritonavir as a therapeutic alternative for COVID-19 \\ El uso de Lopinavir y Ritonavir como alternativa terapéutica al COVID-19
}

Recebido: 25/05/2021 | Revisado: 04/06/2021 | Aceito: 14/06/2021 | Publicado: 28/06/2021

\author{
Eduarda Luiza Maciel da Silva \\ ORCID: https://orcid.org/0000-0003-1757-5559 \\ Universidade Federal da Fronteira Sul, Brasil \\ E-mail: eduardaluizamds@gmail.com \\ Guilherme Vinício de Sousa Silva \\ ORCID: https://orcid.org/0000-0002-3170-6503 \\ Universidade Federal da Fronteira Sul, Brasil \\ E-mail: guilhermemeduffs@gmail.com \\ Graciela Soares Fonseca \\ ORCID: https://orcid.org/0000-0001-9506-0409 \\ Universidade Federal da Fronteira Sul, Brasil \\ E-mail: graciela.fonseca@uffs.edu.br \\ Cesar Andres Diaz Arias \\ ORCID: https://orcid.org/0000-0002-9748-1300 \\ Centro Universitário Claretiano, Brasil \\ E-mail: cesar.andres.arias@gmail.com
}

\begin{abstract}
Resumo
A pandemia causada pelo novo coronavírus (SAR-CoV-2) representa um desafio para a saúde pública do mundo inteiro, em função da alta transmissibilidade do vírus, consequente sobrecarga dos sistemas de saúde e grande número de óbitos. Assim, estudos e pesquisas estão sendo realizados no intuito de identificar alternativas terapêuticas seguras e eficazes para a COVID-19, doença causada pelo SAR-CoV-2, visto que ainda não há um consenso nem evidência científica de que algum medicamento possa suprimir a excreção viral sem trazer massivos efeitos colaterais ao paciente. Nessa perspectiva, alguns fármacos estão sendo utilizados na prática clínica e, dentre eles, encontra-se a combinação do Lopinavir com o Ritonavir, antiretrovirais comumente utilizados no tratamento do HIV. O objetivo dessa revisão narrativa é analisar o uso da combinação Lopinavir/Ritonavir como alternativa terapêutica para a COVID-19. Os resultados indicam que a associação Lopinavir/Ritonavir tem sido utilizada como tratamento coadjuvante nos casos de COVID-19, normalmente combinada com outros medicamentos. Estudos sugerem que essa combinação não interfere no tempo de UTI e que a porcentagem de óbitos não é significativa. Quando aplicada no início dos sintomas, essas medicações causam redução na excreção viral e menor número de intubação. No entanto, há contradição referente a isso, já que alguns estudos sugerem aumento na excreção viral em pacientes que receberam esses medicamentos. Reforça-se a necessidade de intensificar os estudos relacionados ao tratamento para COVID-19 no sentido de gerar dados conclusivos para que as medicações possam ser utilizadas com eficácia e segurança.
\end{abstract}

Palavras-chave: Infecções por Coronavirus; Lopinavir; Ritonavir; Tratamento farmacológico.

\begin{abstract}
Pandemy caused by the new coronavirus (SAR-CoV-2) represents a challenge for public health worldwide, due to the high transmissibility of the virus, consequent overload of health systems and large number of deaths. Studies and research are being carried out in order to identify safe and effective therapeutic alternatives for COVID-19, a disease caused by SAR-CoV-2, since there is still no consensus or scientific evidence that any drug can suppress viral excretion without causing massive side effects to the patient. In this perspective, some drugs are being used in clinical practice and, among them, is the combination of Lopinavir with Ritonavir, antiretroviral drugs commonly used in HIV treatment. The aim of this narrative review is to analyze the use of the Lopinavir/Ritonavir combination as a therapeutic alternative for COVID-19. The results indicate that the Lopinavir/Ritonavir combination has been used as an adjunctive treatment in cases of COVID-19, usually combined with other medications. Studies suggest that this combination does not interfere with ICU time and that the percentage of deaths is not significant. When applied at the onset of symptoms, these medications cause reduced viral excretion and fewer intubations. However, there is a contradiction regarding this, as some studies suggest an increase in viral excretion in patients who received these drugs. It reinforces the need to intensify studies related to treatment for COVID-19 in order to generate conclusive data so that medications can be used effectively and safely.
\end{abstract}

Keywords: Coronavirus infections; Lopinavir; Ritonavir; Drug therapy. 


\begin{abstract}
Resumen
La pandemia provocada por el nuevo coronavirus (SAR-CoV-2) representa un desafío para la salud pública a nivel mundial, debido a la alta transmisibilidad del virus, la consiguiente sobrecarga de los sistemas de salud y la gran cantidad de muertes. Así, se están realizando estudios e investigaciones con el fin de identificar alternativas terapéuticas seguras y efectivas para COVID-19, una enfermedad causada por SAR-CoV-2, ya que aún no existe consenso o evidencia científica de que algún fármaco pueda inhibir la excreción viral. sin causar efectos secundarios masivos al paciente. En esta perspectiva, algunos fármacos se están utilizando en la práctica clínica y, entre ellos, se encuentra la combinación de Lopinavir con Ritonavir, fármacos antirretrovirales comúnmente utilizados en el tratamiento del VIH. El objetivo de esta revisión narrativa es analizar el uso de la combinación Lopinavir / Ritonavir como alternativa terapéutica para COVID-19. Los resultados indican que la combinación de Lopinavir / Ritonavir se ha utilizado como tratamiento adyuvante en casos de COVID-19, generalmente combinado con otros medicamentos. Los estudios sugieren que esta combinación no interfiere con el tiempo en la UCI y que el porcentaje de muertes no es significativo. Cuando se aplican al inicio de los síntomas, estos medicamentos reducen la excreción viral y provocan menos intubaciones. Sin embargo, existe una contradicción al respecto, ya que algunos estudios sugieren un aumento de la excreción viral en los pacientes que recibieron estos fármacos. Refuerza la necesidad de intensificar los estudios relacionados con el tratamiento del COVID-19 con el fin de generar datos concluyentes para que los medicamentos se puedan utilizar de forma eficaz y segura.
\end{abstract}

Palabras clave: Coronavirus infections; Lopinavir; Ritonavir; Quimioterapia.

\title{
1. Introdução
}

Em dezembro de 2019, surgiram em Wuhan, uma província da China, diversos casos de pacientes com uma infecção respiratória acompanhada de pneumonia grave, posteriormente definida como uma nova doença, a Coronavirus Disease (COVID-19), causada por um coronavírus, o SARS-CoV-2 (Coronavírus da Síndrome Respiratória Aguda Grave 2). Em poucos meses, a doença tornou-se uma pandemia e converteu-se no maior obstáculo que a saúde mundial enfrentou em décadas, visto que quando sintomática, a infecção viral é capaz de desencadear quadros graves e potencialmente fatais, manifestando quadro clínico caracterizado pela pneumonia precedida por febre, tosse seca e mialgia (Huang et al., 2020).

Medidas de prevenção do contágio como o distanciamento social e a quarentena foram adotadas em diversos países, objetivando diminuir a curva de infecção com vistas a evitar a sobrecarga dos sistemas de saúde (Caetano et al., 2020). No entanto, a pandemia já dura mais de um ano e os efeitos negativos são evidenciados a cada dia, não só pelo significativo número de mortes decorrentes da COVID-19 mas, pelas consequências econômicas, aumento das desigualdades sociais, incremento na prevalência de doenças crônicas, dentre outros.

Coronavírus são vírus envelopados que possuem um nucleocapsídeo composto por uma fita única de ácido ribonucleico (RNA) senso positivo. Estes vírus são envoltos por proteínas do envelope e de membrana, além de proteínas estruturais com formato de espículas (do inglês Spyke), que são encontradas em todos os coronavírus e lhes conferem uma aparência de coroa. Os coronavírus da subfamília Orthocoronavirinae podem se subdividir em quatro gêneros: alfa, beta, gama e delta, sendo os dois primeiros os causadores de doenças em humanos (Li et al., 2020).

Por meio da análise filogenética dos genomas completos, constatou-se que o SARS-CoV-2 pertence ao gênero Betacoronavírus, uma vez que a identidade de sequência no gene ORF 1ab é menor que 90\%, em comparação com outros vírus pertencentes a esse gênero (Zhu N et al., 2020). O mesmo ocorre com o SARS-CoV, causador da Síndrome Respiratória Aguda Grave (SARS) e o MERS-CoV, responsável pela Síndrome Respiratória do Oriente Médio (MERS).

As doenças virais costumam causar sintomas relacionados ao trato respiratório superior, juntamente com febre, tosse e cefaleia (Li et al., 2020). Entretanto, os vírus SARS-CoV, MERS-CoV e SARS-CoV-2 causam doenças com sintomas diferentes das demais infecções virais, atingindo o trato respiratório inferior (Wu \& Mcgoogan, 2020). Existe, no entanto, grande semelhança entre a fisiopatologia da SARS e da COVID-19: na SARS, há edema e infiltração de células inflamatórias, resultantes de uma resposta inflamatória do organismo, que pode causar infiltração alveolar, além de alargamento e danos do septo alveolar, assim como danos nas paredes das arteríolas intersticiais pulmonares ( $\mathrm{Li}$ et al., 2020); por outro lado, na 
COVID-19, há intensa resposta inflamatória, responsável por induzir hiperplasia de células, edema, formação de membrana hialina, infiltração de células inflamatórias e danos ao tecido pulmonar (Tian et al., 2020).

Essas características, associadas ao alto potencial de transmissibilidade do SARS-CoV-2 e às variantes que surgiram após um ano do início do primeiro surto de COVID-19, foram responsáveis por mais de 176 milhões de casos e mais de 3,8 milhões de mortes no mundo até a segunda semana de junho de 2021. No Brasil, no mesmo período, foram mais de 17 milhões de casos e 480 mil vidas perdidas para a doença (WHO, 2021).

No segundo semestre de 2020, algumas vacinas que vinham sendo desenvolvidas em diferentes países foram aprovadas para uso em massa, representando grande esperança de controle da doença. Elas apresentam altas porcentagens (maiores que 90\%) para proteção relacionada ao agravamento ou morte por causa da doença, no entanto, estudos iniciais demonstraram que os indivíduos vacinados poderiam desenvolver a doença com sintomas leves com porcentagens de eficácia entre 50\% e 75\%. Estudos recentes realizados pelo Centro de Controle e Prevenção de Doenças (CDC), dos Estados Unidos, com vacinas que tem como base RNA mensageiro, demonstrou efetividade de $80 \%$ para pessoas sintomáticas e assintomáticas, após o recebimento da primeira dose da vacina, e de $90 \%$ após a segunda dose (Thompson et al. 2021).

Esse panorama traz esperança mas, reforça que a humanidade continuará convivendo com o SAR-CoV-2 e suas consequências, ainda que em uma dimensão de controle maior do que a existente atualmente. Ou seja, é imperativo investigar possibilidades terapêuticas para tratar as pessoas que desenvolverem COVID-19, evitando agravamento e óbitos.

Frente a isso, pesquisadores estão desenvolvendo estudos diversos com o objetivo de identificar um tratamento seguro e eficaz para tratar a COVID-19. Nesse contexto, alguns medicamentos como Arbidol, Favipiravir, Remdesivir, Cloroquina, Hidroxicloroquina, Azitromicina, Ribavirin, Lopinavir e Ritonavir, dentre outros, foram ou estão sendo testados.

O Lopinavir e o Ritonavir foram considerados pela Organização Mundial da Saúde (OMS) como uma combinação promissora para o tratamento da COVID-19, sendo incluídos em estudos clínicos internacionais, englobados no projeto "Solidarity", lançado pela OMS em 2020, com o objetivo de encontrar tratamentos eficazes contra a doença, por meio de comparações de resultados para quatro diferentes tratamentos (OMS, 2020)

O objetivo desta revisão narrativa é analisar os dados publicados na literatura sobre o uso da combinação Lopinavir/Ritonavir como alternativa terapêutica para a COVID-19.

\section{Mecanismo de Ação do SARS-COV-2}

O SARS-CoV-2 é um Betacoronavírus, assim como o SARS-CoV e o MERS-CoV, entretanto, sua sequência é relativamente diferente dos outros subtipos (Chen et al., 2020), além de possuir uma grande similaridade genética com o coronavírus RaTG13, encontrado em morcegos na província de Yunnan, em 2013 (Zhai et al., 2020). O SARS-CoV-2 possui vírions envelopados, é formado por uma fita única de RNA senso positivo (Xu et al., 2020) e possui a enzima transcriptase reversa sendo, portanto, um retrovírus (Ferreira et al., 2020).

Este vírus apresenta formato de coroa devido às proteínas transmembrana (Spyke), presentes na superfície viral (Chen et al., 2020). Estas proteínas possuem um domínio ligante do receptor, semelhante aos do SARS-CoV (Lu R et al., 2020), sendo um domínio único e contínuo que possui toda a informação necessária para interagir com o receptor da célula do organismo hospedeiro (Letko, Marzi \& Munster, 2020), a fim de possibilitar a entrada nas células por meio da indução da fusão das membranas (Weiss \& Leibowitz, 2011).

Tanto o SARS-CoV-2 quanto o SARS-CoV utilizam a enzima conversora da angiotensina 2 (ECA2) como receptor celular para adentrar na célula hospedeira, por intermédio da ligação do ectodomínio da proteína Spike do vírus com o domínio peptidase da proteína ECA2 (Wrapp et al., 2020) que, por sua vez, está intimamente ligada à maturação da angiotensina e, portanto, controla a vasoconstrição e a pressão arterial (Yan et al., 2020). Após a ligação, a protease da célula hospedeira cliva 
a proteína Spike, liberando o peptídeo de fusão, um processo fundamental para a entrada bem sucedida do vírus (Millet \& Whittaker, 2015). Posteriormente, ocorre a fusão das membranas, liberando o genoma viral no citoplasma da célula do hospedeiro, o que permite a replicação do vírus (Bosch \& Rotier, 2008 apud Millet \& Whittaker, 2020).

Uma parte do RNA viral é traduzido em duas poliproteínas replicases: ppla e pp1ab, que contêm proteínas não estruturais. As poliproteínas são então clivadas pelas proteases PLpro e 3CLpro, liberando essas proteínas não estruturais como componentes individuais e funcionais (Chen, Yiu \& Wong, 2020; Fehr \& Perlman, 2015), formando o complexo replicasetranscriptase: uma vesícula com membrana dupla, na qual vai acontecer os processos de replicação e transcrição (Fehr \& Perlman, 2015; Knoops et al., 2008).

As proteínas não estruturais também vão ser codificadas, formando enzimas importantes nos processos de replicação e transcrição, como a RNA polimerase DNA dependente e helicase (Fehr \& Perlman, 2015). Após esses processos, o vírus realiza a transcrição do seu RNA em DNA complementar, cDNA, que é prontamente incorporado ao DNA do hospedeiro, e assim os novos ciclos celulares terão o DNA viral incorporado à célula do indivíduo e, dessa forma, proteínas virais poderão ser produzidas e causar danos ao organismo (Ferreira et al., 2017).

Com relação à proteína ECA2, ela é muito expressa em células endoteliais de veias e artérias, células do músculo liso arterial, pneumócitos do tipo I e II, enterócitos e células do túbulo proximal. Além disso, ela é pouco expressa em células da mucosa oral e nasal (Hamming et al., 2004). Isso explica diversos sintomas e sinais encontrados em pacientes positivos para COVID-19 e o fato deles estarem pouco relacionados ao trato respiratório superior, como espirro, dor de garganta e escarro, indicando que a proteína ECA2 pode estar expressa em maior quantidade no trato respiratório inferior (Huang et al., 2020). Por estar expressa em maior quantidade em células endoteliais e do músculo liso em praticamente todos os órgãos, o vírus pode facilmente ser levado a todos os tecidos do organismo através da circulação sanguínea (Hamming et al., 2004).

As partículas de SARS-CoV-2 induzem uma cascata de citocina e, por conseguinte, respostas imunes que mudam a conformação de células brancas periféricas, assim como células do sistema imune, como os linfócitos (Chen et al., 2020). Essa cascata se caracteriza como uma resposta inflamatória exacerbada e prolongada, visto que o sistema imunológico do hospedeiro é hiperativado, liberando uma quantidade grande de citocinas, parte fundamental do processo inflamatório (Ragab et al., 2020). Foram identificados níveis aumentados de interleucina 6 (IL-6), interleucina 10 (IL-10) e fator de necrose tumoral alfa (TNF- $\alpha$ ) em pacientes infectados pelo SARS-CoV-2 (Diao et al., 2020).

O aumento dos níveis circulantes de citocina leva a um excessivo infiltrado decorrente da atração de células inflamatórias como neutrófilos e monócitos (Qing Ye, Wang \& Mao, 2020), além de causar uma desestabilização das interações célula-célula, danos a barreira vascular e capilar e dano alveolar difuso, podendo levar a falência de órgãos e morte, devido ao grande infiltrado inflamatório (Shimizu, 2019). No que compete aos danos pulmonares decorrentes da cascata de citocina, podem surgir lesões no tecido pulmonar deixando-o suscetível a uma síndrome (Síndrome do Desconforto Respiratório Agudo - SDRA), podendo acarretar falência dos órgãos e necessidade de ventilação mecânica (Lipworth et al., 2020).

Pesquisadores demonstraram que parte dos pacientes com COVID-19 (35\%) apresentaram linfopenia, que está relacionada à forma de ataque do vírus (Chen et al., 2020). Esta linfopenia pode ser explicada pelo fato de que uma estimulação contínua do vírus pode levar a uma exaustão das células T, o que causaria uma redução da produção de citocinas. Entretanto, isso não acontece na COVID-19, visto que, o número total de células T, CD4+ e CD8+ estão negativamente relacionados aos níveis de TNF- $\alpha$, IL-6 e IL-10, respectivamente. Dessa forma, indica-se que a linfopenia pode também estar relacionada à cascata de citocina ocorrida na referida doença. Os níveis de TNF- $\alpha$ podem estar envolvidos neste fenômeno, uma vez que, este promove a apoptose de células T, assim como o IL-10, que bloqueia a proliferação dessas células. Assim, o estudo sugere que as citocinas na COVID-19 não são secretadas pelas células T, mas sim promovem a sua apoptose e necrose, 
levando à linfopenia (Diao et al., 2020). Diversos pacientes também apresentaram linfopenia e leucopenia e alguns, especialmente os que foram internados em Unidade de Terapia Intensiva (UTI), tiveram maiores níveis de trombina e Ddímero (Huang et al., 2020), indicando alterações hematológicas (Barros et al., 2020).

Como consequência de todos esses processos, surgem os sintomas clínicos da COVID-19, sendo o mais encontrado a febre, presente em 43,8\% dos pacientes na sua admissão e em 88,7\% após hospitalização. O segundo sintoma mais comum é a tosse $(67,8 \%)$ e os menos comuns são a náusea/vômito (5\%) e a diarreia (3,8\%) (Guan et al., 2020). Em relação aos padrões de tomografia computadorizada de tórax, os achados mais comuns foram opacidade em vidro fosco e sombras bilaterais irregulares. Microscopicamente, notou-se, no tecido pulmonar, danos às células epiteliais alveolares, espessamento intersticial e formação de cartilagem hialina e componentes do dano alveolar difuso. Há, ainda, necrose fibrinóide de pequenos vasos, congestão vascular, além de um grande infiltrado de células inflamatórias. No tecido cardíaco, observou-se edema, fibrose intersticial e hipertrofia do miocárdio (Tian et al., 2020). Por fim, no fígado de uma paciente internada com COVID-19, verificou-se esteatose microvesicular e pouca atividade lobular e portal, podendo ser causadas pelo SARS-CoV-2 ou pelos medicamentos utilizados no controle da doença (Xu et al., 2020).

\section{Lopinavir e Ritonavir}

No tratamento para o HIV, o Lopinavir e o Ritonavir inibem a clivagem das poliproteínas GAG e GAG-pol, mediada pela protease do HIV-1, resultando em vírions imaturos não infecciosos do HIV (Tomasselli \& Heinrikson, 2000).

Quando analisados de mamenira indepentende, o Lopinavir se mostra mais eficiente que o Ritonavir para diminuir a carga viral de HIV no hospedeiro nos estudos in vitro, porém demonstra baixa biodisponibilidade nos estudos in vivo (Lu C et. $a l$, 2020). Já o Ritonavir, além de atuar na protease do vírus, também inibe o citocromo p450 3A4 (CYP3A4) (Kempf et al., 1997), enzima responsável pela metabolização do Lopinavir.

Em relação a isso, uma pesquisa comprovou que os níveis de ABT-378, um potente inibidor da protease do HIV, quando administrado com Ritonavir, foram altos e constantes (Sham et al., 1998), o que sugere que a associação Lopinavir/Ritonavir prolongue a biodisponibilidade do Lopinavir no organismo.

A protease 3CLpro realiza um papel fundamental no processo clivagem das poliproteínas, permitindo assim a replicação viral. Dessa forma, o Lopinavir torna-se uma opção de tratamento, visto que é um inibidor de protease aspártica. Entretanto, a estrutura molecular desta protease não é a mesma da 3CLpro, uma vez que essa é uma cisteíno protease, podendo afetar a eficácia terapêutica do medicamento (Wang et al., 2020).

\subsection{Lopinavir e Ritonavir na SARS e MERS}

Os vírus SARS-CoV, MERS-CoV e SARS-CoV-2 possuem muitas similaridades genéticas e estruturais. As doenças originadas por eles (SARS, MERS e COVID-19) apresentam algumas características em comum, como o mesmo receptor celular e uma fisiopatogenia semelhante (Li et al., 2020). O Lopinavir e o Ritonavir já foram utilizados e testados para a SARS e para a MERS, o que torna válido avaliar os resultados destes estudos para que possamos compreender melhor o interesse de testar a eficácia terapêutica destes antirretrovirais na COVID-19.

Primeiramente utilizado no tratamento para a SARS, o Ritonavir foi estudado como um inibidor da protease 3CL, entretanto, ele não mostrou atividade em uma concentração de $50 \mu \mathrm{M}$. O mesmo estudo testou o Lopinavir, constatando que a

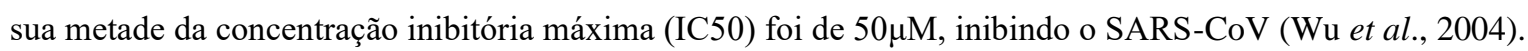

Um estudo constatou que nem o Lopinavir, nem o Ritonavir mostraram efeitos inibitórios na replicação do SARSCoV (Yamamoto et al., 2004). Uma análise da ligação da protease principal do SARS-CoV com o Lopinavir revelou que 
metade do medicamento é deixado de fora do sítio catalítico, além de inferir que a eficácia do Lopinavir pode ser baixa (Zhang \& Yap, 2004).

Outro trabalho observou dois grupos de pacientes com idade média de 42 anos, em que 44 deles receberam a combinação Lopinavir/Ritonavir como tratamento inicial, 31 como terapia de resgate e todos tinham confirmação microbiológica de infecção por SARS CoV. Esses dados foram comparados com um grupo que não recebeu tratamento com Lopinavir/Ritonavir. No grupo que recebeu Lopinavir/Ritonavir como terapia inicial, observou-se que 68,2\% dos pacientes tiveram episódios de baixa saturação e 27,3\% precisaram receber Metilprednisolona, enquanto no grupo que não foi tratado com Lopinavir/Ritonavir, esses dados foram de 84,5\% e 55,4\%, respectivamente (Chan et al., 2003).

Um estudo demonstrou, a partir da análise da dinâmica molecular entre Lopinavir/Ritonavir e a enzima livre 3CLpro do SARS-CoV, que essas duas drogas se ligam com eficácia ao sítio ativo de ligação da proteína viral inibindo-a, o que sugere sua eficácia inibitória, visto que esta enzima é uma proteína fundamental no processo de replicação e funcionamento do vírus (Nukoolkarn et al., 2008).

Por fim, um estudo a respeito do uso de Lopinavir/Ritonavir, no tratamento de SARS, demonstrou que o desenvolvimento de SDRA ou morte em 21 dias foi, significativamente, menor no grupo que recebeu a combinação Lopinavir/Ritonavir, em comparação ao grupo controle. Outra constatação, deste mesmo estudo, foi que o Lopinavir a 4 $\mu \mathrm{g} / \mathrm{mL}$, em combinação com Ribavirin a $50 \mu \mathrm{g} / \mathrm{mL}$, reduziu o efeito citopatogênico do SARS-CoV in vitro (Chu et al., 2004).

Com relação à MERS, após a administração de Lopinavir/Ritonavir, Ribavirina e IFN-ALFA2a peguilado, em uma paciente diagnosticada com MERS, a febre começou a cair e, após dois dias, cessou. Após 6 dias do início do tratamento com antivirais, não foi mais constatado carga viral na paciente (Kim et al., 2016). Pesquisadores estudaram a ação de Lopinavir/Ritonavir, Mofetil Micofenolato (MMF) e Interferon- $\beta 1 \mathrm{~b}$ em saguis infectados com MERS. Entre os grupos que receberam Lopinavir/Ritonavir e interferon- $\beta 1 \mathrm{~b}$, não foi constatado o desenvolvimento de sintomas graves, com raio-x de tórax normal, além de menor mortalidade em comparação com o grupo que recebeu MMF e que não foram tratados. Em consonância com estes achados, as lesões no tecido pulmonar foram mais brandas no grupo que recebeu Lopinavir/Ritonavir em comparação com os outros, além de ter tido a menor carga viral média no tecido pulmonar e renal. Por fim, os autores inferem que o tratamento da MERS com Lopinavir/Ritonavir melhorou as características clínicas, radiológicas e patológicas da doença (Chan et al., 2015)

O Lopinavir inibiu a replicação do MERS-CoV, in vitro, com um EC50s de $8 \mu \mathrm{M}$, e um efeito protetor máximo ( $89 \%$ de inibição) foi observado a uma dose de $12 \mu \mathrm{M}$. Sugere-se que o Lopinavir tenha como alvo a protease principal (Mpro) do vírus ou que ele bloqueie algum outro alvo que se encontra na pós-entrada do vírus no ciclo replicativo. Nesse estudo, os autores classificam o Lopinavir como um dos medicamentos promissores que devem ser estudados posteriormente, visto que a concentração necessária para inibir o vírus in vitro pode ser alcançada também no organismo humano (Wilde et al., 2014).

Por fim, um estudo acompanhou o caso de uma paciente com MERS que recebeu Lopinavir/Ritonavir, Interferon peguilado e Ribavirin a partir do $13^{\circ}$ dia de doença. Dois dias após o início do tratamento, não foi mais constatada viremia, entretanto, a presença de RNA viral nas secreções respiratórias persistiu até a quarta semana da doença e, posteriormente, a paciente foi a óbito (Spanakis et al., 2014). Outro estudo não constatou atividade in vitro do Lopinavir contra o MERS-CoV (Chan J et al., 2013).

\subsection{Lopinavir e Ritonavir na COVID-19}

A associação Lopinavir/Ritonavir é administrada no combate ao HIV na posologia de $400 \mathrm{mg} / 100 \mathrm{mg}$, respectivamente, a cada 12 horas (Porche, 2001). Essa mesma dose foi utilizada para tratamento da infecção viral por SARS- 
CoV, obtendo resultados positivos (Chu et al., 2004). Entretanto, a eficácia de Lopinavir/Ritonavir em relação ao novo coronavírus tem se mostrado divergente na literatura.

Um estudo randomizado realizado com 199 pacientes com COVID-19, sendo 100 tratados com o tratamento padrão e 99 com a combinação Lopinavir/Ritonavir, concluiu que os pacientes que receberam Lopinavir/Ritonavir não apresentaram diferença no tempo de melhora clínica em relação ao outro grupo. Além disso, a mortalidade aos 28 dias foi similar nos dois grupos e a ocorrência de eventos gastrointestinais, como náusea, vômito e diarreia esteve mais presente nos pacientes que receberam os antirretrovirais. No entanto, o tempo de estadia na UTI foi menor no grupo que recebeu a combinação de medicamentos e o número de pacientes com complicações graves, como insuficiência renal e infecção secundária, ou que demandaram ventilação mecânica invasiva ou não invasiva por insuficiência respiratória, foi menor no grupo que recebeu Lopinavir/Ritonavir. O estudo concluiu que não há benefício significativo do uso de Lopinavir/Ritonavir em pacientes com SARS-CoV-2, quando comparado ao tratamento padrão, visto que a carga viral não foi reduzida em um menor tempo em comparação com o outro grupo (Cao et al., 2020).

Outra pesquisa constatou que esses medicamentos não reduziram a excreção viral em pacientes com pneumonia leve, já que o aumento nos valores de cycle threshold (ct), por dia, entre o grupo que recebeu Lopinavir/Ritonavir e o grupo em tratamento padrão foram, respectivamente, 0,9 e 1,0. A ocorrência de eventos adversos como SDRA ou morte dentro de 21 dias foi menor no grupo que recebeu Lopinavir/Ritonavir, além de uma redução no desenvolvimento de infecções hospitalares. Os autores não recomendam a administração destes medicamentos visto a imprecisão dos dados (Cheng et al., 2020).

Ye et. al (2020) defendem a aplicação de Lopinavir/Ritonavir como tratamento coadjuvante em pacientes com a COVID-19 que tenham contraído pneumonia, devido aos efeitos benéficos como redução da temperatura corporal e restauração dos mecanismos fisiológicos normais, sem efeitos tóxicos ou colaterais evidentes, além de uma duração média de dias menor para testar negativo para SARS-CoV-2, em comparação ao grupo que não recebeu Lopinavir/Ritonavir. Os autores inferem que a inserção de Lopinavir/Ritonavir no tratamento pode reduzir valores anormais em exames bioquímicos, como leucócitos, linfócitos e proteína c-reativa.

Os benefícios do uso de Lopinavir/Ritonavir foram reforçados em outro trabalho, visto que, após a administração dos medicamentos, a carga viral dos pacientes começou a decair, além de ter sido observado uma melhora nos sintomas clínicos. Entretanto, os autores explicitam que este fato pode ter ocorrido em função do curso natural da doença, e não especificamente pelo uso desses medicamentos. Por fim, inferem que o Lopinavir, em combinação com o Ritonavir, pode ser recomendado para pacientes em grupos de risco com pneumonia em estágio inicial causada pela COVID-19, porém, é de fundamental necessidade que sejam realizados pesquisas e estudos que concluam de forma concisa estas afirmações (Lim et al., 2020).

Uma pesquisa mostrou que a média de duração da excreção viral no grupo que recebeu Lopinavir/Ritonavir entre os 10 primeiros dias do início dos sintomas foi menor, comparado aos outros que começaram o tratamento após os 10 dias. A duração média desse fator, no grupo que não recebeu Lopinavir/Ritonavir, não foi significativamente diferente quando comparado ao grupo que começou o tratamento após os 10 primeiros dias do início dos sintomas. Ainda sobre isso, foi constatado que o uso de Lopinavir/Ritonavir não inibe completamente a replicação do SARS-CoV-2, sendo que o grupo que recebeu Lopinavir/Ritonavir, nos primeiros dias, teve uma duração média de viral shedding de 19 dias (Yan D et al., 2020). Um estudo randomizado controlado constatou que, entre 29 pacientes que receberam o Lopinavir/Ritonavir, a duração média da excreção viral foi de 22 dias, revelando que não houve redução após a administração destes medicamentos (Zhou et al., 2020).

Um relatório observacional buscou comparar o uso de um tratamento padrão, Hidroxicloroquina e Lopinavir/Ritonavir, em pacientes com COVID-19 internados em UTI. Não foi encontrada uma diferença significativa entre os três grupos em relação ao tempo sem necessidade de ventilação, em 28 dias, e também de mortalidade entres os dias 14 e 18 . 
Houve uma redução da carga viral semelhante entre os três grupos, além de a proporção de pacientes com carga viral negativa no sétimo dia também ter sido semelhante (Lecronier et al., 2020).

O relato de uma série de casos dos 18 primeiros pacientes confirmados com COVID-19, em 4 hospitais da Singapura, afirmou que $60 \%$ dos pacientes que receberam Lopinavir/Ritonavir tiveram uma redução na necessidade de oxigênio suplementar em até 3 dias, e 40\% tiveram uma carga viral nula até 2 dias do início da administração dos medicamentos. Entretanto, dois pacientes tiveram insuficiência respiratória progressiva. Em relação aos eventos adversos, foi constatada a presença de náuseas, vômitos e/ou diarreia em 4 dos 5 pacientes tratados com Lopinavir/Ritonavir, além de função hepática anormal em 3 pacientes. Os autores concluíram que a combinação Lopinavir/Ritonavir não foi capaz de prevenir a progressão da doença em 2 pacientes e perceberam que a diminuição da carga viral obtida pelos esfregaços nasofaríngeos foram semelhantes àqueles não tratados com Lopinavir/Ritonavir (Young et al., 2020).

A eficácia terapêutica do Lopinavir/Ritonavir também foi comparada com o Arbidol em um estudo em que um grupo recebeu Lopinavir/Ritonavir na dose de 400/100mg, duas vezes por dia, durante uma semana, e o outro recebeu 0,2g de Arbidol, três vezes ao dia. Não foi constatada diferença na duração da febre e no padrão encontrado nas tomografias de tórax entre os dois grupos. Os pacientes do grupo Lopinavir/Ritonavir tiveram uma maior contagem de neutrófilos e proteína creativa, além de linfopenia e 44,1\% deles apresentaram carga viral após 14 dias da admissão. No grupo que recebeu Arbidol, essa taxa foi nula, sugerindo que o uso de Arbidol é mais efetivo do que o uso da combinação de Lopinavir/Ritonavir (Zhu $\mathrm{Z} e t$ al., 2020).

O Arbidol também foi analisado em uma investigação que buscou avaliar a eficácia do Lopinavir/Ritonavir, de modo isolado ou em combinação com esse medicamento. Os autores constataram que, após 14 dias, o vírus não foi detectado em 94\% dos pacientes do primeiro grupo, enquanto no segundo, este resultado prevaleceu para 53\%. Houve uma melhora nos exames de tomografia de tórax em $69 \%$ dos pacientes do grupo combinado, enquanto no grupo da monoterapia, essa melhora foi constatada em 29\% dos participantes (Deng et al., 2020).

Outro trabalho investigou dois grupos de pacientes, somando 10 participantes, sendo o primeiro tratado com Lopinavir e Interferon $\alpha 2 \mathrm{~b}$ e o segundo apenas com Lopinavir. A opacidade observada em tomografias de tórax apresentou melhora em 4 pacientes que receberam Lopinavir e interferon $\alpha 2 b$. Entretanto, após receberem a terapia combinada, 3 pacientes relataram diarreia e vômito, além de hipocalemia. Os autores inferiram que o uso de Lopinavir foi efetivo e os efeitos adversos encontrados devem ser um fator a ser considerado antes da administração desses medicamentos (Liu et al., 2020).

Um dos fatores que dificultam a real conclusão sobre a eficácia do Lopinavir/Ritonavir é o fato destes serem utilizados em consonância com outros medicamentos. Pesquisadores realizaram um estudo em que pacientes foram escolhidos aleatoriamente para receber Lopinavir/Ritonavir, Ribavirin e Interferon beta-1b, enquanto outro grupo recebeu apenas Lopinavir/Ritonavir. Os resultados constataram que, no grupo que recebeu a combinação tripla, houve supressão da excreção viral, além de menor concentração de IL-6, interleucina importante na cascata de citocina. A melhora clínica foi superior no grupo combinado, com um tempo significativamente menor até o completo alívio dos sintomas em comparação com o outro grupo, sendo de 4 e 8 dias, respectivamente, o que refletiu diretamente em menor tempo de internação hospitalar no primeiro grupo. O estudo concluiu que a combinação Lopinavir/Ritonavir mostrou efeitos similares a um placebo, no que tange à redução da carga viral, sendo portanto, menos seguro e eficiente, além de concluir que um tratamento precoce com a combinação tripla de antivirais é apropriado, visto que, a carga viral do SARS-CoV-2 atinge seu pico logo no início dos sintomas (Hung et al., 2020). 


\section{Considerações Finais}

A associação Lopinavir/Ritonavir tem sido utilizada como tratamento coadjuvante nos casos de COVID-19, usualmente combinados com outros medicamentos. No entanto, a conclusão da análise da eficácia do Lopinavir/Ritonavir torna-se difícil, visto que, como mostrado nesta revisão, não há consenso na literatura sobre isso.

Os resultados encontrados nesta revisão narrativa, sugerem que o uso de Lopinavir/Ritonavir não contribuiu para reduzir os tempos de internação na UTI. Em vários dos estudos realizados, a porcentagem de mortalidade não foi estatisticamente significativa, no entanto, um dos estudos demonstrou que sintomas como náuseas, vômito e diarreia aumentaram nos pacientes tratados com esta associação.

Alguns estudos afirmaram que, quando a associação Lopinavir/Ritonavir foi realizada no tratamento inicial ou nos primeiros dias da infecção (10 dias), os pacientes apresentaram redução da excreção viral, uma grande porcentagem dos pacientes não precisou ser entubada. Além disso, a mortalidade foi menor, quando comparada ao grupo de pacientes que não foram tratados desta forma. A partir disso, é possível inferir que o uso de Lopinavir/Ritonavir só seria útil nas etapas iniciais da infecção, porém, mais estudos são necessários para comprovar essa hipótese.

Outros estudos revelaram resultados contrários em relação à excreção viral, descrevendo que o uso de Lopinavir/Ritonavir não inibiu completamente a replicação do vírus e, dessa forma, não diminuiu a excreção viral, no entanto, novos estudos precisam ser realizados neste sentido sendo que existem muitas contradições entre as diferentes pesquisas realizadas.

As associações com outros medicamentos como a hidroxicloriquina, procedimentos padrões e a associação Lopinavir/Ritonavir, não demonstrou nenhum efeito significativo, ou seja, quando utilizado como tratamento padrão, nem a hidroxicloroquina, nem o Lopinavir/Ritonavir demonstraram diminuir a carga viral ou a porcentagem de mortalidade entre os paciente tratados.

Por outro lado, a associação Lopinavir/Ritonavir, ao ser comparada com o Arbitol, demonstrou que os pacientes tratados com o Arbitol apresentaram redução da carga viral após 14 dias e em uma grande porcentagem dos pacientes, o vírus não foi detectado. Estes resultados indicam que o Arbidol poderia se tornar um novo objeto de pesquisas para o tratamento para COVID-19, e estudos poderiam ser direcionados nesse sentido.

Reforça-se a necessidade de intensificar os estudos relacionados ao tratamento para COVID-19 no sentido de gerar dados conclusivos para que as medicações possam ser utilizadas com eficácia e segurança.

\section{Referências}

Barros, B. C. S., Maia, A. B., Marques, M. A., Prette-Junior, P. R., Fiorelli, S. K. A. \& Cerqueira, F. C. (2020). The role of Angiology and Vascular Surgery in the COVID-19 pandemic. Rev. Col. Bras. Cir., 47, 1-9.

Caetano, R., Silva, A. B., Guedes, A. C. C. M., Paiva, C. C. N., Ribeiro, G. R., Santos, D. L. \& Silva, R. M. (2020). Desafios e oportunidades para telessaúde em tempos da pandemia pela COVID-19: uma reflexão sobre os espaços e iniciativas no contexto brasileiro. Cadernos de Saúde Pública, 36 (5), 1-16.

Cao, B., Wang, Y., Wen, D., Liu, W., Wang, J., Fan, G., Ruan, L., Song, B., Cai, Y., Wei, M., Li, X., Xia, J., Chen, N., Xiang, J., Yu, T., Bai, T., Xie, X., Zhang, L., Li, C., Yuan, Y., Chen, H., Li, H., Huang, H., Tu, S., Gong, F., Liu, Y., Wei, Y., Dong, C., Zhou, F., Gu, X., Xu, J., Liu, Z., Zhang, Y., Li, H., Shang, L., Wang, K., Li, K., Zhou, X., Dong, X., Qu, Z., Lu, S., Hu, X., Ruan, S., Luo, S., Wu, J., Peng, L., Cheng, F., Pan, L., Zou, J., Jia, C., Wang, J., Liu, X., Wang, S., Wu, X., Ge, Q., He, J., Zhan, H., Qiu, F., Guo, L., Huang, C., Jaki, T., Hayden, F. G., Horby, P. W., Zhang, D. \& Wang, C. (2020). A Trial of Lopinavir-Ritonavir in Adults Hospitalized with Severe Covid-19. New England Journal of Medicine, 382 (19),1787-1799.

Chan, J. F. W., Chan, K. H., Kao, R. Y. T., To, K. K. W., Zheng, B. J., Li, C. P. Y., Li, P. T. W., Dai, J., Mok, F. K. Y., Chen, H., Hayden, F. G. \& Yuen, K. Y. (2013). Broad-spectrum antivirals for the emerging Middle East respiratory syndrome coronavirus. J Infect, 67 (6), $606-616$.

Chan, J. F. W., Yao, Y., Yeung, M. L., Deng, W., Bao, L., Jia, L., Li, F., Xiao, C., Gao, H., Yu, P., Cai, J. P., Chu, H., Zhou, J., Chen, H., Qin, C. \& Yuen, K. Y. (2015). Treatment With Lopinavir/Ritonavir or Interferon- $\beta 1 b$ Improves Outcome of MERS-CoV Infection in a Nonhuman Primate Model of Common Marmoset. J Infect Dis, 212 (12), 1904-1913. 
Chan, K. S., Lai, S. T., Chu, C. M., Tsui, E., Tam, C. Y., Wong, M. M. L., Tse, M. W., Que, T. L., Peiris, J. S. M., Sung, J., Wong, V. C. W. \& Yuen, K. Y (2003). Treatment of severe acute respiratory syndrome with lopinavir/ritonavir: a multicentre retrospective matched cohort study. Hong Kong Med J, 9 (6), $399-406$.

Chen, N., Min, Z., Dong, X., Jieming, Q., Gong, F., Han, Y., Qiu, Y., Wang, J., Liu, Y., Wei, Y., Xia, J., Yu, T., Zhang, X. \& Zhang, L. (2020). Epidemiological and clinical characteristics of 99 cases of 2019 novel coronavirus pneumonia in Wuhan, China: a descriptive study. The Lancet, 395, 507-513.

Chen, Y. W., Yiu, C. P. B. \& Wong, K. Y. (2020). Prediction of the SARS-CoV-2 (2019-nCoV) 3C-like protease (3CL) structure: virtual screening reveals velpatasvir, ledipasvir, and other drug repurposing candidates. F1000Res, 9 (129).

Cheng, C. Y., Lee, Y. L., Chen, C. P., Lin, Y. C., Liu, C. E., Liao, C. H. \& Cheng, S. H. (2020). Lopinavir/ritonavir did not shorten the duration of SARS CoV-2 shedding in patients with mild pneumonia in Taiwan. Journal of Microbiology, Immunology and Infection, 53 (3), 488-492.

Chu, C. M., Cheng, V. V. C., Hung, I. F. N., Wong, M. M. L., Chan, K. H., Chan, K. S., Kao, R. Y. T., Poon, L. L. M., Wong, C. L. P., Guan, Y., Peiris, J. S. M. \& Yuen, K. Y. (2004). Role of lopinavir/ritonavir in the treatment of SARS: initial virological and clinical findings. Thorax, 59(3), $252-256$.

Deng, L., Li, C., Zeng, Q., Liu, X., Li, X., Zhang, H., Hong, Z. \& Xia, J. (2020). Arbidol combined with LPV/r versus LPV/r alone against Corona Virus Disease 2019: A retrospective cohort study. J Infect, 81(1), 1-5.

Diao, B., Wang, C., Tan, Y., Chen, X., Liu, Y., Ning, L., Chen, L., Min, L., Liu, Y., Wang, G., Yuan, Z., Feng, Z., Zhang, Y., Wu, Y. \& Chen, Y. (2020). Reduction and Functional Exhaustion of T Cells in Patients With Coronavirus Disease 2019 (COVID-19). Frontiers in Immunology, 11 (827), 1-7.

Fehr, A. R. \& Perlman S. (2015). Coronaviruses: an overview of their replication and pathogenesis. Methods Mol Biol, 1282, 1-23.

Ferreira, B., Izar, F., Lemos, C. \& Ribas, J. L. C. (2017). Antibióticos e antirretrovirais: uma abordagem biotecnológica. Rev. Saúde e Desenvolvimento, 11 (9), 234-248.

Guan, W., Ni, Z., Hu, Y., Liang, W., Ou, C., He, J., Liu, L., Shan, H., Lei, C., Hui, D. S. C., Du, B., Li, L., Zeng, G., Yuen, K. Y., Chen, R., Tang, C., Wang, T., Chen, P., Xiang, J., Li, S, Wang, J. L., Liang, Z. J., Peng, Y. X., Wei, L., Liu, Y., Hu, Y.H., Peng, P., Wang, J. M., Liu, J. Y., Chen, Z., Li, G., Zheng, Z. J., Qiu, S. Q., Luo, J., Ye, C.J., Zhu, S. Y. \& Zhong, N. S. (2020). Clinical Characteristics of Coronavirus Disease 2019 in China. The New England Journal of Medicine, 382(18), 1708-1720.

Hamming, I., Timens, W., Bulthuis, M. L. C., Lely, A. T., Navis, G. J. \& Goor, H. V. (2004). Tissue distribution of ACE2 protein, the functional receptor for SARS coronavirus. A first step in understanding SARS pathogenesis. J Pathol, 203, 631-637.

Huang, C., Wang, Y., Li, X., Ren, L., Zhao, J., Hu, Y., Zhang, L., Fan, G., Xu, J., Gu, X., Cheng, Z., Yu, T., Xia, J., Wei, Y., Wu, W., Xie, X., Yin, W., Li, H., Liu, M., Xiao, Y., Gao, H., Guo, L., Xie, J., Wang, G., Jiang, R., Gao, Z., Jin, Q., Wang, J. \& Cao, B. (2020). Clinical features of patients infected with 2019 novel coronavirus in Wuhan, China. The Lancet, 395(10223), 497-506.

Hung, I. F.N., Lung, K.C., Tso, E. Y.K., Liu, R., Chung, T. W.-H., Chu, M.-Y., Ng, Y.-Y., Lo, J., Chan, J., Tam, A. R., Shum, H.-P., Chan, V., Wu, A. K.-L., Sin, K.-M., Leung, W.-S., Law, W.-L., Lung, D. C., Sin, S., Yeung, P., Yip, C. C.-Y, Zhang, R.R, Fung, A.Y.-F., Yan, E.Y.-W., Leung, K.-H., Daniel, J., Chu, A.W.-H., Chan, W.-M., Ng, A.C.-K., Lee, R., Fung, K., Yeung, A., Wu, T.-C., Chan, J.W.-M., Yan, W.-W., Chan, W.-M., Chan, J.F.-W., Lie, A.K.-W., Tsang, O.T.-Y., Cheng, V.C.-C., Que, T.-L., Lau, C.-S., Chan, K.-H., To, K. K.-W. \& Yuen, K.-Y. (2020). Triple combination of interferon beta-1b, lopinavir-ritonavir, and ribavirin in the treatment of patients admitted to hospital with COVID-19: an open-label, randomised, phase 2 trial. The Lancet, 395(10238), 1695-1704

Kempf, D. J., Marsh, K. C., Kumar, G., Rodrigues, A. D., Denissen, J. F., McDonald, E., Kukulka, M. J., Hsu, A., Granneman, G. R., Baroldi, P. A., Sun, E., Pizzuti, D., Plattner, J. J., Norbeck, D. W. \& Leonard, J. M. (1997). Pharmacokinetic enhancement of inhibitors of the human immunodeficiency virus protease by coadministration with ritonavir. Antimicrob Agents Chemother, 41(3), 654-660.

Kim, U. J., Won, E. J., Kee, S. J., Jung, S. I. \& Jang, H. C. (2016). Combination therapy with lopinavir/ritonavir, ribavirin and interferon- $\alpha$ for Middle East respiratory syndrome. Antivir Ther, 21(5), 455-459.

Knoops, K., Kikkert, M., Worm, S. H. E., Dobbe, J. C. Z., Meer, Y., Koster, A. J., Mommaas, A. M. \& Snijder, E. J. (2008). SARS-Coronavirus Replication Is Supported by a Reticulovesicular Network of Modified Endoplasmic Reticulum. PLoS Biol, 6(9), 1957-1974.

Lecronier, M., Beurton, A., Burrel, S., Haudebourg, L., Deleris, R., Le Marec, J., Virolle, S., Nemlaghi, S., Bureau, C., Mora, P., De Sarcus, M., Clovet, O., Duceau, B., Grisot, P. H., Pari, M. H., Arzoine, J., Clarac, U., Boutolleau, D., Raux, M., Delemazure, J., Faure, M., Decavele, M., Morawiec, E., Mayaux, J., Demoule, A. \& Dres, M. (2020). Comparison of hydroxychloroquine, lopinavir/ritonavir, and standard of care in critically ill patients with SARS-CoV-2 pneumonia: an opportunistic retrospective analysis. Critical Care, 24(1), 418.

Letko, M., Marzi, A. \& Munster, V. (2020). Functional assessment of cell entry and receptor usage for SARS-CoV-2 and other lineage B betacoronaviruses. Nature Microbiology, 5, 562-569

Li, G., Fan, Y., Lai, Y., Han, T., Li, Z., Zhou, P., Pan, P., Wang, W., Hu, D., Liu, X., Zhang, Q. \& Wu, J. (2020). Coronavirus infections and immune responses. J Med Virol, 92(4), 424-432.

Lim, J., Jeon, S., Shin, H. Y., Kim, M. J., Seong, Y. M., Lee, W. J., Choe, K. W., Kang, Y. M., Lee, B. \& Park, S. J. (2020). Case of the Index Patient Who Caused Tertiary Transmission of Coronavirus Disease 2019 in Korea: the Application of Lopinavir/Ritonavir for the Treatment of COVID-19 Pneumonia Monitored by Quantitative RT-PCR. J Korean Med Sci, 35(6), e79.

Lipworth, B., Chan, R., Lipworth, S. \& Kuo, C. R. (2020). Weathering the Cytokine Storm in Susceptible Patients with Severe SARS-CoV-2 Infection. The Journal of Allergy and Clinical Immunology: In Practice, 8(6), 1798-1801.

Liu, F., Xu, A., Zhang, Y., Xuan, W., Yan, T., Pan, K., Yu, W. \& Zhang, J. (2020). Patients of COVID-19 may benefit from sustained Lopinavir-combined regimen and the increase of Eosinophil may predict the outcome of COVID-19 progression. International Journal of Infectious Diseases, 95, $183-191$. 
Lu, C. C., Chen, M. Y., Lee, W. S. \& Chang, Y. L. (2020). Potential therapeutic agents against COVID-19: What we know so far. Journal of the Chinese Medical Association, 83(6), 534-536.

Lu, R., Zhao, X., Li, J., Niu, P., Yang, B., Wu, H., Wang, W., Song, H., Huang, B., Zhu, N., Bi, Y., Ma, X., Zhan, F., Wang, L., Hu, T., Zhou, H., Hu, Z., Zhou, W., Zhao, L., Chen, J., Meng, Y., Wang, J., Lin, Y., Yuan, J., Xie, Z., Ma, J., Liu, W.J., Wang, D., Xu, W., Holmes, E.C., Gao, G.F., Wu, G., Chen, W., Shi, W. \& Tan, W. (2020). Genomic characterisation and epidemiology of 2019 novel coronavirus: implications for virus origins and receptor binding. The Lancet, 395(10224).

Millet, J. K. \& Whittaker, G. R. (2015). Host cell proteases: Critical determinants of coronavirus tropism and pathogenesis. Virus Research, 202, 120-134.

Nukoolkarn, V., Lee, V. S., Malaisree, M., Aruksakulwong, O. \& Hannongbua, S. (2008). Molecular dynamic simulations analysis of ritonavir and lopinavir as SARS-CoV 3CLpro inhibitors. Journal of Theoretical Biology, 254(4), 861-867.

Panagopoulos, P., Petrakis, V., Panopoulou, M., Trypsianis, G., Penlioglou, T., Pnevmatikos, I. \& Papazoglou, D. (2020). Lopinavir/ritonavir as a third agent in the antiviral regimen for SARS-CoV-2 infection. Journal of Chemotherapy, 33(3), 193-197.

Porche, D. J. (2001). Lopinavir/Ritonavir. Journal of the Association of Nurses in AIDS Care, 12(2), 101-104.

Ragab, D., Salah, E.H., Taeimah, M., Khattab, R. \& Salem, R. (2020). The COVID-19 Cytokine Storm, What We Know So Far. Frontiers in Immunology, 11.

Sham, H. L., Kempf, D. J., Molla, A., Marsh, K. C., Kumar, G. N., Chen, C. M., Kati, W., Stewart, K., Lal, R., Hsu, A., Betebenner, D., Korneyeva, M., Vasavanonda, S., McDonald, E., Saldivar, A., Wideburg, N., Chen, X., Niu, P., Park, C. \& Jayanti, V. (1998). ABT-378, a Highly Potent Inhibitor of the Human Immunodeficiency Virus Protease. Antimicrobial Agents and Chemotherapy, 42(12), 3218-3224.

Spanakis, N., Tsiodras, S., Haagmans, B. L., Raj, V. S., Pontikis, K., Koutsoukou, A., Koulouris, N. G., Osterhaus, A. D. M. E., Koopmans, M. P. G. \& Tsakris, A. (2014). Virological and serological analysis of a recent Middle East respiratory syndrome coronavirus infection case on a triple combination antiviral regimen. International Journal of Antimicrobial Agents, 44(6), 528-532.

Thompson, M.G., Burgess, J. L., Naleway, A. L., Tyner, H. L., Yoon, S. K., Meece, J., Olsho, L. E. W., Caban-Martinez, A. J., Fowlkes, A., Lutrick, K., Kuntz, J. L., Dunnigan, K., Odean, M. J., Hegmann, K. T., Stefanski, E., Edwards, L. J., Schaefer-Solle, N., Grant, L., Ellingson, K., Groom, H. C., Zunie, T., Thiese, M. S., Ivacic, L., Wesley, M. G., Lamberte, J. M., Sun, X., Smith, M. E., Phillips, A. L., Groover, K. D., Yoo, Y. M., Gerald, J., Brown, R. T., Herring, M. K., Joseph, G., Beitel, S., Morrill, T. C., Mak, J., Rivers, P., Harris, K. M., Hunt, D. R., Arvay, M. L., Kutty, P., Fry, A. M., Gaglani, M. (2021) Interim Estimates of Vaccine Effectiveness of BNT162b2 and mRNA-1273 COVID-19 Vaccines in Preventing SARS-CoV-2 Infection Among Health Care Personnel, First Responders, and Other Essential and Frontline Workers - Eight U.S. MMWR Morb Mortal Wkly, 70(13):495-500.

Tian, S., Xiong, Y., Liu, H., Niu, L., Guo, J., Liao, M. \& Xiao, S. Y. (2020). Pathological study of the 2019 novel coronavirus disease (COVID-19) through postmortem core biopsies. Modern Pathology, 33(6), 1007-1014.

Tomasselli, A. G. \& Heinrikson, R. L. (2000). Targeting the HIV-protease in AIDS therapy: a current clinical perspective. Biochimica et Biophysica Acta (BBA) - Protein Structure and Molecular Enzymology, 1477(1-2), 189-214.

Wang, Q., Zhao, Y., Chen, X. \& Hong, A. (2020). Virtual screening of approved clinic drugs with main protease (3CLpro) reveals potential inhibitory effects on SARS-CoV-2. Journal of Biomolecular Structure and Dynamics, 1-11.

Weiss, S. R. \& Leibowitz, J. L. (2011). Coronavirus Pathogenesis. Advances in Virus Research, 85-164

Wilde, A. H., Jochmans, D., Posthuma, C. C., Zevenhoven-Dobbe, J. C., van Nieuwkoop, S., Bestebroer, T. M., van den Hoogen, B. G., Neyts, J. \& Snijder, E. J. (2014). Screening of an FDA-Approved Compound Library Identifies Four Small-Molecule Inhibitors of Middle East Respiratory Syndrome Coronavirus Replication in Cell Culture. Antimicrobial Agents and Chemotherapy, 58(8), 4875-4884.

World Health Organization (2020). "Solidarity" clinical trial for COVID-19 treatments. https://www.who.int/emergencies/diseases/novel-coronavirus2019/global-research-on-novel-coronavirus-2019-ncov/solidarity-clinical-trial-for-covid-19-treatments.

Wrapp, D., Wang, N., Corbett, K. S., Goldsmith, J. A., Hsieh, C. L., Abiona, O., Graham, B. S. \& McLellan, J. S. (2020). Cryo-EM structure of the 2019nCoV spike in the prefusion conformation. Science, 367(6483), 1260-1263.

Wu, C. Y., Ja, J. T., Ma, S. H., Kuo, C. J., Juan, H. F., Cheng, Y. S., E Hsu, H. H., Huang, H. C., Wu, D., Brik, A., Liang, F. S., Liu, R. S., Fang, J. M., Chen, S. T., Liang, P. H. \& Wong, C. H. (2004). Small molecules targeting severe acute respiratory syndrome human coronavirus. Proceedings of the National Academy of Sciences, 101(27), 10012-10017.

Wu, Z. \& McGoogan, J. M. (2020). Characteristics of and Important Lessons From the Coronavirus Disease 2019 (COVID-19) Outbreak in China. JAMA, $323(13), 1239$.

Xu, Z., Shi, L., Wang, Y., Zhang, J., Huang, L., Zhang, C., Liu, S., Zhao, P., Liu, H., Zhu, L., Tai, Y., Bai, C., Gao, T., Song, J., Xia, P., Dong, J., Zhao, J. \& Wang, F.S. (2020). Pathological findings of COVID-19 associated with acute respiratory distress syndrome. The Lancet Respiratory Medicine, 8(4), 420-422.

Xu, X., Chen, P., Wang, J., Feng, J., Zhou, H., Li, X., Zhong, W. \& Hao, P. (2020). Evolution of the novel coronavirus from the ongoing Wuhan outbreak and modeling of its spike protein for risk of human transmission. Science China Life Sciences, 63(3), 457-460.

Yamamoto, N., Yang, R., Yoshinaka, Y., Amari, S., Nakano, T., Cinatl, J., Rabenau, H., Doerr, H. W., Hunsmann, G., Otaka, A., Tamamura, H., Fujii, N. \& Yamamoto, N. (2004). HIV protease inhibitor nelfinavir inhibits replication of SARS-associated coronavirus. Biochemical and Biophysical Research Communications, 318(3), 719-725.

Yan, D., Liu, X.Y., Zhu, Y., Huang, L., Dan, B., Zhang, G. \& Gao, Y. (2020). Factors associated with prolonged viral shedding and impact of lopinavir/ritonavir treatment in hospitalised non-critically ill patients with SARS-CoV-2 infection. European Respiratory Journal, 56(1), 2000799. 
Research, Society and Development, v. 10, n. 7, e43410716505, 2021

(CC BY 4.0) | ISSN 2525-3409 | DOI: http://dx.doi.org/10.33448/rsd-v10i7.16505

Yan, R., Zhang, Y., Li, Y., Xia, L., Guo, Y. \& Zhou, Q. (2020). Structural basis for the recognition of SARS-CoV-2 by full-length human ACE2. Science, $367(6485), 1444-1448$.

Ye, Q., Wang, B. \& Mao, J. (2020). The pathogenesis and treatment of the 'Cytokine Storm' in COVID-19. Journal of Infection, 80(6), 607-613.

Ye, X. T., Luo, Y. L., Xia, S. C., Sun, Q. F., Ding, J. G., Zhou, Y., Chen, W., Wang, X. F., Zhang, W. W., Du, W. J., Ruan, Z. W. \& Hong, L. (2019). Clinical efficacy of lopinavir/ritonavir in the treatment of Coronavirus disease 2019. European Review for Medical and Pharmacological Sciences, 24(6).

Young, B.E., Ong, S.W.X., Kalimuddin, S., Low, J. G., Tan, S. Y., Loh, J., Ng, O.T., Marimuthu, K., Ang, L.W., Mak, T.M., Lau, S.K., Anderson, D.E., Chan, K. S., Tan, T.Y., Ng, T.Y., Cui, L., Said, Z., Kurupatham, L., Chen, M.I.C. \& Chan, M. (2020). Epidemiologic Features and Clinical Course of Patients Infected With SARS-CoV-2 in Singapore. JAMA, 323(15), 1488.

Zhang, X.W. \& Yap, Y. L. (2004). Old drugs as lead compounds for a new disease? Binding analysis of SARS coronavirus main proteinase with HIV, psychotic and parasite drugs. Bioorganic \& Medicinal Chemistry, 12(10), 2517-2521.

Zhai, P., Ding, Y., Wu, X., Long, J., Zhong, Y. \& Li, Y. (2020). The epidemiology, diagnosis and treatment of COVID-19. International Journal of Antimicrobial Agents, 55(5), 105955.

Zhou, F., Yu, T., Du, R., Fan, G., Liu, Y., Liu, Z., Xiang, J., Wang, Y., Song, B., Gu, X., Guan, L., Wei, Y., Li, H., Wu, X., Xu, J., Tu, S., Zhang, Y., Chen, H. \& Cao, B. (2020). Clinical course and risk factors for mortality of adult inpatients with COVID-19 in Wuhan, China: a retrospective cohort study. The Lancet, 395(10229), 1054-1062.

Zhu, N., Zhang, D., Wang, W., Li, X., Yang, B., Song, J., Zhao, X., Huang, B., Shi, W., Lu, R., Niu, P., Zhan, F., Ma, X., Wang, D., Xu, W., Wu, G., Gao, G. F. \& Tan, W. (2020). A Novel Coronavirus from Patients with Pneumonia in China, 2019. New England Journal of Medicine, 382(8), 727-733.

Zhu, Z., Lu, Z., Xu, T., Chen, C., Yang, G., Zha, T., Lu, J. \& Xue, Y. (2020). Arbidol monotherapy is superior to lopinavir/ritonavir in treating COVID-19. Journal of Infection, 81(1), e21-e23. 